\title{
Random acts of innovation: Infiltrating silos and creating an interprofessional culture for quality care
}

Everyone has a story. One I vividly recall was told to me by a mother of a medically compromised twoyear-old boy whose life literally depended on the multiple therapies and medical procedures he received daily. "I'm never alone in my home" she told me. "But I'm grateful for the care that keeps Emmett ${ }^{1}$ alive." Given the constant presence of providers in her life, I asked if there was anything that she thought they should know or perhaps didn't understand about parents' needs. Without hesitation she replied, "They've got to communicate with each other. It shouldn't be a parent's job to coordinate their activities. We have enough to do to care for our sick children. My advice - make sure that you work with people willing to make the time to listen to you and to each other. You need to be a team. It makes all the difference."

This special edition of WORK is dedicated to people like the mother described above who need us to be prepared with requisite attitudes and skills that make all the difference. Over the last three decades health organizations have urged educators, practitioners, administrators, and policy-makers to integrate interprofessional competencies, including cross-disciplinary communication, into academic programming and service delivery systems [1-5]. Such recommendations have been spurred in part by the increasingly complex and chronic health needs of people who come to be our clients and patients. Mounting concerns about patient safety and medical errors have furthermore generated national and international calls to investigate and improve quality of care for people throughout the life course. Guiding

\footnotetext{
${ }^{1}$ The child's name has been deidentified to protect his privacy.
}

principles for interprofessional education and practicefirst and foremost place the patient and family at the center of care. Knowing the ethics and responsibilities of other practitioners and honoring their respective roles adds value to collaborative practice irrespective of profession and regardless of workplace setting. Lastly communicative competencies promote compassionate practices across professional fields and prepare emerging practitioners for effective team-based work. In combination these guidelines are thought to better position practitioners to ensure favorable outcomes.

Within the pages of this special edition are writings generated by champions of interprofessional education and workplace practice. Reeves, Tassone, Parker and Wagner, colleagues from the University of Toronto, the vortex of much IPE research and innovation, place IPE in historical context and provide an overview of current and evolving developments in the field. Their reporthelps readers to better understand the challenges faced by educators and practitioners to develop and implement truly interprofessional programming in their institutions.

Along with Reeves et al.'s report, many of the articles contained in this edition utilize the Centre for the Advancement of Interprofessional Education's (CAIPE)definition of IPE as "when two or more professions learn with, from and about each other to improve collaboration and the quality of care" [6]. Similarly, interprofessional practice is understood as an active collaboration between two or more professions aimed at optimizing person and family-centered care. This definition distinguishes interprofessional practices from the more commonly utilized forum of multidisciplinary practice as it emphasizes communication and collabo- 
ration between practitioners in tandem with the people and families who become our patients/clients. Interprofessional practice is also differentiated from other collaborative models as team leadership is determined by the needs of clients/patients rather than predetermined by professional status or a one-size-fits-all formula for service delivery.

Konrad and Browning's [9] thought piece invites readers to situate interprofessionality within a broader framework of relational and caring practice. The authors contend that twenty-first century health care suffers disconnection from caring traditions that are at the heart of theories for practice across health professions. As they see it, contemporary health educators are challenged to dually prepare students with relational competencies to both care for future clients/patients and to function as thoughtful and effective interprofessional team members because no single profession can effect change alone.

Integration of interprofessional competencies into academic classroom and field experiences are well represented byauthors who themselves are educators, practitioners and researchers. Deutschland, Suter, and Lait, collaborators from the Health System and Workforce Research Unit at the Alberta Health Services in Calgary, report on their use of the "interprofessional enhancement approach". This approach to IPE shared learningeffectively avoids the often-cited obstacles to integrating interprofessional curriculum and internships. The brilliant design feature of this method is in its assimilation of interprofessional learning opportunities into existing coursework and placement experiences.

Gray and MacRae, Sheldon, Cavanaugh, Croninger, Osgood, Robnett, Seigle, and Simonsen, and Willis, Lloyd, and Jenkins add richness to the issue's offerings by describing IPE field innovations created in collaboration with community partners. These represent ongoing opportunities for students across disciplines to participate in shared learning in several key areas, for example fall prevention with occupational therapy (OT), nursing, and social work students; acute care provision in hospital settings with OT and physical therapy students, and outreach preventative screenings to assess for hypertension provided by undergraduate health professions students in an underserved community. All of these articles describe IPE endeavors not only aimed at integrated student learning but also designed, executed, and evaluated by cross-disciplinary faculty and community partners. Thus each project not only models interprofessionality in action but also provides examples of how to gently transform institutional culture away from exclusively siloed curriculum and fieldwork.
Engagement of consumers as members of the IPE instructional team is a common theme in MacRae's and Cavanaugh and Konrad's papers. Both describe transformative learning that takes place when consumers share their lived knowledge with learners. The Interprofessional Geriatric Education Program (IGEP) at the University of New England conceived in 1996 and currently situated in the physician assistant department, has as its centerpiece elder teachers who serve as members of the instructional team. According to MacRae, the student/faculty make-up of IGEP has evolved over the years but its principles remain rooted in relationship-centered care. Cavanaugh and Konrad, a PT and social work faculty respectively, report on a three-year collaborative project that highlights the experiences and needs ofpeople with acquired disabilities. Like IGEP, this shared learning experience for PT, social work, and nursing students includes consumers as co-instructors. The authors found that both students and consumer instructors highly valued the experience of learning with and from each other.

Transformational learning is also the focus of Morton's article, which illustrates the author's emerging interprofessional and transcultural learning model. Transcultural Immersion in Healthcare (TIH) takes place in an urban setting in the country of Ghana, West Africa where twice a year students participate in a range of learning, clinical and reflective activities within a context of intensive cultural immersion. According to Morton, TIH advances transcultural and interprofessional learning experiences for students and faculty while simultaneously building collaborative partnerships with community health workers and local healthcare services. Morton's work coincides with the World Health Organization's impetus to educate students to practice from a position of global collaboration [4]. As noted in WHO's recent statement on interprofessional health practice "Once students understand how to work interprofessionally, they are ready to enter the workplace as a member of the collaborative practice team" [4].

It has been my pleasure to work with and learn from diverse colleagues passionate in their desire to improve the health, functioning and care of people by way of improving communication and collaboration in our work settings. My co-author David Browning introduced me to the following Japanese proverb: none of us is as smart as all of us. I agree. Given the crisis we face in health care today it's time for us to collectively sidestep the cynicism of the current health care divide and find ways to work together to enhance the quality of care for all and ensurehealth as a human right. 
Guest Editor

Shelley Cohen Konrad PhD, LCSW School of Social Work, and InterProfessional

Education Collaborative

University of New England

716 Stevens Avenue

Portland, ME 04103, USA

Tel: +1 207-797-7688 x4501

E-mail: scohenkonrad@une.edu

\section{References}

[1] Institute of Medicine (2001). Crossing the quality chasm: A new health system for the 21 st century. Washington, D. C.: National Academies Press.
[2] Institute of Medicine (2003). Health Professions Education: A Bridge to Quality. Washington, D. C.: National Academies Press.

[3] Institute of Medicine (2010). Redesigning continuing education in the health professions, Committee on planning a continuing health profession education institute, Washington, D. C.: National Academies Press.

[4] World Health Organization (2010). Framework for Action on Interprofessional Education and Collaborative Practice. Available at: http://whqlibdoc.who.int/hq/2010/WHO_HRH_HPN_ 10.3_eng.pdf.

[5] Interprofessional Education Collaborative (2011). Core competencies for Interprofessional Collaborative Practice. Available at: http://www.aacom.org/InfoFor/educators/mec/ipskilldev/ ipe/Documents/CCrpt05-10-11.pdf.

[6] Centre for the Advancement of Interprofessional Education (2010). Interprofessional Education: A Definition. Available at: www. caipe.org.uk/about-us/defining-ipe. 